\title{
Impact of $\beta 2-1$ fructan on faecal community change: results from a placebo-controlled, randomised, double-blinded, cross-over study in healthy adults
}

\author{
Sandra T. Clarke ${ }^{1}$, Stephen P. J. Brooks ${ }^{2}$, G. Douglas Inglis ${ }^{3}$, L. Jay Yanke $^{3}$, Judy Green ${ }^{2}$, \\ Nicholas Petronella ${ }^{4}$, D. Dan Ramdath ${ }^{5}$, Premysl Bercik ${ }^{6}$, Julia M. Green-Johnson ${ }^{1}$ and Martin Kalmokoff * \\ ${ }^{1}$ Applied Bioscience Graduate Program, Faculty of Science, University of Ontario Institute of Technology, Oshawa, ON, \\ Canada, $L 1 H 7 K 4$ \\ ${ }^{2}$ Bureau of Nutrition Research, Health Canada, Ottawa, ON, Canada, K1A OKO \\ ${ }^{3}$ Lethbridge Research Centre, Agriculture and Agri-Food Canada, Lethbridge, AB, Canada, T1J 4B1 \\ ${ }^{4}$ Bureau of Food Surveillance and Science Integration, Health Canada, Ottawa, ON, Canada, K1A OKO \\ ${ }^{5}$ Guelph Food Research and Development Centre, Agriculture and Agri-Food Canada, Guelph, ON, Canada, N1G 5C9 \\ ${ }^{6}$ Gastroenterology, Department of Medicine, McMaster University, Hamilton, Ontario, Canada, L8S $4 L 8$ \\ ${ }^{7}$ Atlantic Food and Horticulture Research Centre, Agriculture and Agri-Food Canada, Kentville, NS, Canada, B4N 1J5
}

(Submitted 1 March 2017 - Final revision received 19 July 2017 - Accepted 31 July 2017 - First published online 28 September 2017)

\section{Abstract}

Healthy adults ( $n$ 30) participated in a placebo-controlled, randomised, double-blinded, cross-over study consisting of two $28 \mathrm{~d}$ treatments ( $\beta 2-1$ fructan or maltodextrin; $3 \times 5 \mathrm{~g} / \mathrm{d}$ ) separated by a $14-\mathrm{d}$ washout. Subjects provided $1 \mathrm{~d}$ faecal collections at days 0 and 28 of each treatment. The ability of faecal bacteria to metabolise $\beta 2-1$ fructan was common; eighty-seven species (thirty genera, and four phyla) were isolated using anaerobic medium containing $\beta 2-1$ fructan as the sole carbohydrate source. $\beta 2-1$ fructan altered the faecal community as determined through analysis of terminal restriction fragment length polymorphisms and $16 \mathrm{~S}$ rRNA genes. Supplementation with $\beta 2-1$ fructan reduced faecal community richness, and two patterns of community change were observed. In most subjects, $\beta 2-1$ fructan reduced the content of phylotypes aligning within the Bacteroides, whereas increasing those aligning within bifidobacteria, Faecalibacterium and the family Lachnospiraceae. In the remaining subjects, supplementation increased the abundance of Bacteroidetes and to a lesser extent bifidobacteria, accompanied by decreases within the Faecalibacterium and family Lachnospiraceae. $\beta 2-1$ Fructan had no impact on the metagenome or glycoside hydrolase profiles in faeces from four subjects. Few relationships were found between the faecal bacterial community and various host parameters; Bacteroidetes content correlated with faecal propionate, subjects whose faecal community contained higher Bacteroidetes produced more caproic acid independent of treatment, and subjects having lower faecal Bacteroidetes exhibited increased concentrations of serum lipopolysaccharide and lipopolysaccharide binding protein independent of treatment. We found no evidence to support a defined health benefit for the use of $\beta 2-1$ fructans in healthy subjects.

Key words: B2-1 Fructan: Bifidobacteria: Faecal community change: Cultivation analysis

The colon hosts a complex bacterial community which provides a barrier to resist colonisation by pathogens, metabolises both dietary and endogenous substrates providing energy to the host in the form of SCFA, and plays a role in the development and maintenance of immunity ${ }^{(1)}$. It is widely stated that manipulating gut communities using diet can provide health benefits ${ }^{(1-3)}$. For example, feeding lactulose to patients with chronic liver disease improves health by reducing blood ammonia concentrations ${ }^{(4,5)}$ by increasing the demand for endogenous ammonia as a $\mathrm{N}$ source for gut bacteria ${ }^{(6)}$. Similarly, the prebiotic concept also claims that by selectively stimulating the growth of colonic bifidobacteria host health can be improved ${ }^{(7)}$. To date, there is little evidence supporting any specific health benefit for prebiotics in healthy subjects ${ }^{(8-12)}$, in contrast to emerging evidence concerning the negative effects of including rapidly fermented oligosaccharides in the diets of patients with a variety of preexisting gut conditions ${ }^{(13,14)}$.

Fructan prebiotics are polymers of fructose linked by a $\beta 2-1$ glycosidic bond. These carbohydrates are found in grains, fruits and vegetables ${ }^{(15)}$ where they function as plant storage polymers $^{(16)}$, and constitute a portion of daily fibre intake ${ }^{(17)}$. As the $\beta 2-1$ bond is resistant to cleavage in the small intestine, these pass into the colon where they are metabolised by the gut bacteria. Feeding $\beta 2-1$ fructans increases the abundance of

Abbreviations: LBP, lipopolysaccharide binding protein; T-RFLP, terminal restriction fragment length polymorphism.

* Corresponding author: M. Kalmokoff, email martin.kalmokoff@agr.gc.ca 
bifidobacteria in human faeces ${ }^{(7-9,12,18,19)}$. Purported benefits from promoting bifidobacteria growth include stimulation of host immunity ${ }^{(20)}$, suppression of pathogens ${ }^{(21,22)}$, decreased intestinal transit times ${ }^{(23)}$, as well as positive impacts on various pathologies $^{(24-26)}$ and host physiology ${ }^{(27-29)}$. Although clinical trials involving healthy subjects have confirmed the bifidogenic effect, they have failed to demonstrate any specific health link associated with this change $\mathrm{e}^{(8-11,30)}$ and report only modest effects on immune activity ${ }^{(31)}$. The most consistently observed impacts associated with $\beta 2-1$ fructan supplementation in healthy subjects are increased flatulence, various forms of intestinal discomfort, and a loosening of the stool ${ }^{(8-11,18,32)}$. Feeding $\beta 2-1$ fructan may also reduce concentrations of branched-chain fatty acids and other secondary metabolites arising from protein fermentation in the colon ${ }^{(9,33)}$, as well as increase faecal butyrate outputs ${ }^{(9,26,29)}$

Although the bifidogenic effect of $\beta 2-1$ fructan is well documented, it is often claimed to be a selective effect ${ }^{(7)}$. Much less is known concerning the impacts of $\beta 2-1$ fructan on the other bacterial species present within the faecal community. These polymers are metabolised by additional species isolated from faeces $^{(34-36)}$, and more recent work has also noted effects on altering Faecalibacterium and Bacteroidetes levels in human faeces following the ingestion of $\beta 2-1$ fructan ${ }^{(12,19)}$. Moreover, not only does the extent of the bifidogenic effect vary between individuals $^{(37)}$, but the response has also been observed following the feeding of other rapidly fermented oligosaccharides ${ }^{(37-40)}$.

Previously, we reported on the impacts of $\beta 2-1$ fructan supplementation on immunological, physiological and psychological parameters across this cohort ${ }^{(9)}$. Here, we report the effects of $\beta 2-1$ fructan supplementation on global change within these faecal bacterial communities and on the impacts of these changes on a range of host immunological and physiological parameters.

\section{Methods}

\section{Study design}

Subject recruitment, clinical orientation, written informed consent, enrolment, allocation, randomisation and blinding were undertaken as previously described ${ }^{(9)}$. Inclusion criteria for the trial were participants aged $18-50$ years, in good general health, and a BMI of $18-30 \mathrm{~kg} / \mathrm{m}^{2}$. Subjects taking medication or any health supplement, regular ingestion of high $\beta 2-1$ fructan containing food products, known gastrointestinal diseases, food allergies, or intolerance to any ingredient in the treatments were excluded from the trial. There were thirty subjects who enrolled and completed both phases of the study (seventeen women and thirteen men). This trial was conducted in accordance with the guidelines laid down in the Declaration of Helsinki. All procedures were approved by the research ethics boards at Health Canada, McMaster University, University of Ontario Institute of Technology, and by the Canadian SHIELD Ethics Review Board. The study is registered as 'Effects of fructan prebiotics on the intestinal microbiota' at www.ClinicalTrials. gov (clinical trial identifier NCT01277445). The clinical trial spanned from 1 November 2010 to 5 April 2011 and was carried out at Nutrasource Diagnostics Inc.. Results assessing the impacts of $\beta 2-1$ fructan on immunology, physiology and wellbeing across this cohort have previously been published ${ }^{(9)}$.

In brief, the study used a randomised, placebo-controlled, double-blinded, cross-over design where each subject supplemented their usual diet with a commercially available $\beta 2-1$ fructan (Orafti ${ }^{\circledR}$ Synergy1 (BENEO), 50:50 mixture of inulin and short chain oligosaccharides) or placebo (maltodextrin). Subjects ingested $15 \mathrm{~g} / \mathrm{d}$ total of each supplement $(3 \times 5 \mathrm{~g}$ ingested with each meal). Treatments were $28 \mathrm{~d}$ in duration, with a $14-\mathrm{d}$ wash-out between supplement phases (Fig. 1). Faecal samples were collected at the beginning (day 0) and at the end (day 28) of each supplement phase, for a total of four collections per subject over the $70-\mathrm{d}$ trial. Individual samples were stored at $-20^{\circ} \mathrm{C}$ until processed. Repeated freeze-thaw of faecal samples was avoided; frozen subsamples of faeces were removed for distribution to team members for processing. Immediately before processing, the subsample was thawed, mixed to obtain homogenous slurries and weighed (approximately $1.0 \mathrm{~g}$ for each analysis).

Review of the daily diaries kept by individual subjects over the duration of the clinical trial revealed that three subjects were prescribed oral antibiotics during the clinical trial. Antibiotics have been shown to impact faecal communities ${ }^{(41)}$ and affected diversity of the faecal communities of these subjects (results not shown). It was also revealed that a fourth subject suffered from constipation, and consumed laxatives and an over-the-counter intestinal cleansing product for the duration of the trial. The faecal community of this individual was found to be more distantly related with time independent of treatment (results not shown). These four subjects were included in the analysis of cultivable faecal bacteria (Fig. $1 ; n$ 30), but not in assessments of faecal bacterial community change across the cohort (Fig. 1; $n$ 26).

\section{Cultivation-based analysis}

Faecal processing and cultivation was undertaken in an anaerobic chamber with an atmosphere of $\mathrm{CO}_{2}: \mathrm{H}_{2}(90: 10 \% \mathrm{v} / \mathrm{v})$. Faecal samples were weighed and recorded (about $1.0 \mathrm{~g}$ ), blended using a stomacher in $9 \mathrm{ml}$ of an anaerobic dilution solution $^{(42)}$, and then serially diluted using the same solution. Aliquots from the dilution series were plated onto a modified L-10 agar $^{(43)}$ containing $\beta 2-1$ fructan (Inulin, $2 \% \mathrm{w} / \mathrm{v}$; Sigma Chemical Co.) as the sole carbohydrate source. Cultures were incubated at $37^{\circ} \mathrm{C}$ for $72-96 \mathrm{~h}$. The plated dilutions yielding 30-300 colony-forming units (CFU) were sampled, and 25-50 colonies were randomly selected and transferred to L-10 broth. Occasionally, colonies exhibiting an unusual colony morphology (i.e. from a separate dilution used to enumerate CFU) were also transferred onto L-10. Emphasis was placed on sampling all colony morphologies (two to six morphologies per faecal sample). Subcultures were maintained at $37^{\circ} \mathrm{C}$ for $48 \mathrm{~h}$ and streaked onto modified L-10 agar to confirm purity. Pure isolates were grown in L-10 liquid medium and stored as frozen glycerol $(15 \%, \mathrm{v} / \mathrm{v})$ stocks $\left(\right.$ at $\left.-80^{\circ} \mathrm{C}\right)$. Isolates were identified by a combination GC analysis of the cellular fatty acids ${ }^{(44)}$ (MIDI, Inc.), and by sequencing of near full length $16 \mathrm{~S}$ rRNA genes. 


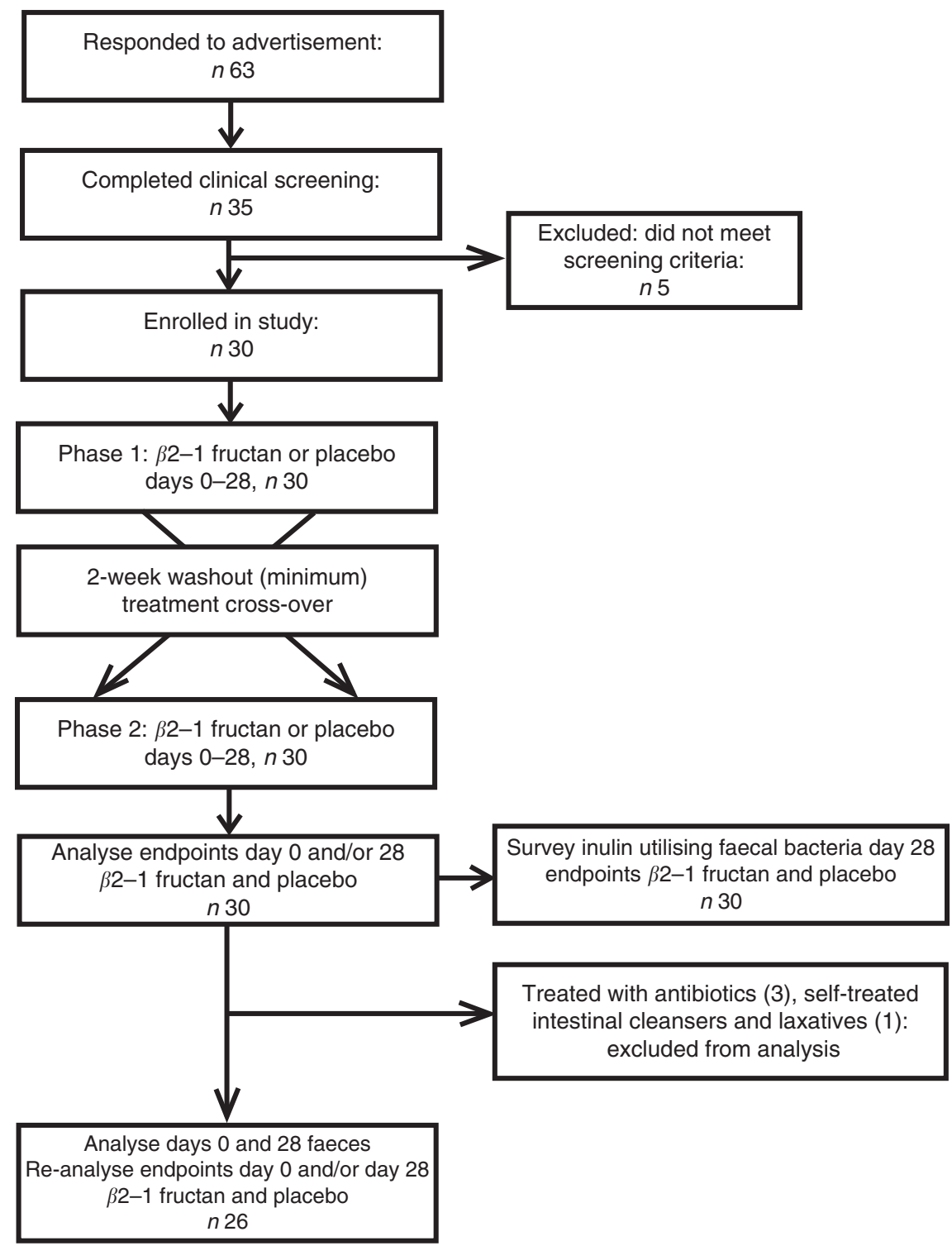

Fig. 1. Consolidated Standard of Reporting Trials diagram: progress of subjects through the trial.

\section{Isolation of faecal DNA}

Faecal community DNA was isolated by first freeze grinding samples in liquid $\mathrm{N}_{2}$ as previously described ${ }^{(45)}$. DNA was purified using the Qiagen faecal DNA isolation kit according to the manufacturer's procedure for difficult to lyse bacteria and was isolated from two sub-samplings of each homogenised $1 \mathrm{~d}$ faecal collection. Isolated community DNA was quantified by spectrophotometry and stored frozen at $-20^{\circ} \mathrm{C}$.

\section{Terminal restriction fragment length polymorphism community analysis}

Terminal restriction fragment length polymorphism (T-RFLP) analysis of faecal communities was completed using the following primers based on 16S rDNA; 6-fam-44f, 5'-6-fam-GAGTTTGATCMTGGCTCAG-3' (IDT) and VIC-1492r, 5'-VIC-GGYTACCTTGTTACGACTT-3', HPLC purified (Life
Technologies). The forward primer was modified ${ }^{(45)}$ from its original design ${ }^{(46)}$. The forward primer was labelled with carboxyfluorescein (FAM) and the reverse primer was labelled with VIC. Each PCR reaction was prepared in triplicate, with each $20 \mu \mathrm{l}$ reaction containing $10 \mathrm{ng}$ community DNA $(2 \mu \mathrm{l}), 1 \mathrm{X}$ PCR buffer, $0 \cdot 2 \mathrm{~mm}$ deoxynucleotides (dNTP), $0 \cdot 2 \mu \mathrm{m}$ forward primer (6-fam 27f), $0 \cdot 2 \mu \mathrm{m}$ reverse primer (VIC-1492r), $0 \cdot 1 \mu \mathrm{g} / \mu \mathrm{l}$ bovine serum albumin, 0.5 U HotStar Taq Plus (Qiagen) and nuclease free water. PCR conditions were as follows: $5 \mathrm{~min}$ at $95^{\circ} \mathrm{C}$ for one cycle, twenty-five cycles at $94^{\circ} \mathrm{C}$ for $30 \mathrm{~s}, 53^{\circ} \mathrm{C}$ for $1 \mathrm{~min} 30 \mathrm{~s}$ and $72^{\circ} \mathrm{C}$ for $1 \mathrm{~min}$, and one cycle at $72^{\circ} \mathrm{C}$ for $10 \mathrm{~min}$. The $3-$ $20 \mu \mathrm{l}$ reactions were pooled and unincorporated primer and nucleotides removed using the PCR cleanup kit (Bio Basic). Cleaned PCR product was re-suspended in $50 \mu \mathrm{l}$ nuclease free water. PCR products were first visualised on a $1 \%$ Tris-acetateEDTA buffer (TAE) gel and quantified by comparing to a standardised $100 \mathrm{bp}$ DNA ladder (Promega), then $75 \mathrm{ng}$ of PCR product were digested at $55^{\circ} \mathrm{C}$ for $1 \mathrm{~h}$ in a $25 \mu \mathrm{l}$ reaction 
containing $12.5 \mu \mathrm{l}$ MaeIII incubation buffer (Roche), $3 \mathrm{U}$ of MaeIII and nuclease free water. Samples were precipitated by adding $2 \mu \mathrm{l}$ of $3 \mathrm{~m}$ sodium acetate ( $\mathrm{pH} 5.2$ ) and $50 \mu \mathrm{l}$ of $95 \%$ ethanol, and maintaining the mixture at $-20^{\circ} \mathrm{C}$ for $20 \mathrm{~min}$. Samples were immediately centrifuged for $20 \mathrm{~min}$ at maximum speed, and the supernatant was removed. Pellets were washed with $500 \mu \mathrm{l}$ of $70 \%$ ethanol, centrifuged for $5 \mathrm{~min}$, and supernatants removed. Pellets were allowed to dry completely, and resuspended in 9.25 $\mu \mathrm{l} \mathrm{Hi-Di} \mathrm{Formamide} \mathrm{(Life} \mathrm{Technologies)} \mathrm{and}$ $0 \cdot 25 \mu \mathrm{l}$ ROX $500 \mathrm{XL}$ size standard (Life Technologies). Samples were denatured for $3 \mathrm{~min}$ at $95^{\circ} \mathrm{C}$, chilled on ice, and loaded onto an ABI 3130 genetic analyzer (Life Technologies). Samples were then separated using a $36 \mathrm{~cm}$ column and POP7 matrix (Life Technologies). All samples were run in duplicate, but only terminal restriction fragments that were common to both runs were analysed (i.e. one composite T-RFLP profile per sample).

T-RFLP profiles were exported and peaks sized using Genemapper 4.0 software (Life Technologies) with the Local Southern size calling method (Applied Biosystems) as described previously $^{(47)}$. Composite data was exported to T-REX ${ }^{(48)}$, noise was subtracted and then a binary matrix was constructed. Euclidean distance between samples was calculated and principal component analysis (PCA) was applied (PRIMER-E Ltd). Statistical significance among groups was determined in PRIMER-E using permutational ANOVA ${ }^{(49)}$. Analysis of bacterial community change was undertaken using Permanova using the Primer 7 analysis package (Primer-E Ltd). Permanova assumes equal variances between groups.

\section{5 rRNA gene sequencing and analysis}

Faecal community DNA samples were subjected to tag-encoded FLX amplicon sequencing ( 454 Life Sciences; Roche) undertaken by Research Testing Laboratory. Nucleotide bases 27-519 (relative to the Escherichia coli 16S rRNA gene) were targeted using the forward primer F44 and the reverse primer 519R and conditions as previously described $^{(50)}$. Sequence files ranged from 4000 to 20000 reads/sample. In files containing $>5000$ reads, 5000 sequences were randomly sampled using Mothur ${ }^{(51)}$. Files containing $<5000$ reads were processed in their entirety. DNA was isolated from two separate samplings (technical replicates) of each 1d faecal collection, both of which were sequenced. Sequence analysis was carried out previously described ${ }^{(50)}$. Sequences derived from each individual faecal sample were initially binned using ESPRIT ${ }^{(52)}$ using a cut-off which resulted in approximately 200 operational taxonomic units (OTU). OTU contained in each sample were checked for chimeras using Chimera-slayer implemented through Mothur ${ }^{(51)}$, with suspect sequences removed. Sequences were aligned against the Silva data base ${ }^{(53)}$, and further binned into their closest matching phylotype. Subsequently, sequences were aligned using ClustalW, a nearest neighbour tree was generated using SeaView ${ }^{(54)}$, and sequences falling within a 3\% sequence divergence were binned together. Final sequences for each individual sample were then classified using Seq-match program available at the Ribosomal Database Project $(\mathrm{RDP})^{(55)}$. Sequences occurring less than three times in each data set or having an RDP coefficient of similarity of $<0.8$ were removed from further analysis. Samples from each individual were processed as a group then combined to identify shared phylotypes across all thirty subjects. Phylotype abundances in replicate faecal samples were averaged, before analysis.

\section{Metagenomic analysis}

Community DNA from four randomly selected subjects over the course of the trial was subjected to a shotgun-based metagenomic analysis. Library preparation and sequencing was carried out using standard protocols as recommended by the manufacturer (Illumina Inc.) and carried out at Genome Quebec. All raw reads were first used in a Blastn against the National Center for Biotechnology Information (NCBI) non-redundant nucleotide database. Each read was then binned based on its corresponding hit's GenInfo Identifier (GI) number. The resulting bins, containing numerous reads, were then assembled into contigs with SPAdes version 3.6.0. Each contiguous sequence was used in a Blastx against the NCBI non-redundant amino acid database where the resulting Blastx reports were used as input for MEGAN ${ }^{(56)}$ and assigned to SEED (http://www.theseed.org) functional groups. Contiguous DNA sequences were also searched against the Carbohydrate-Active enZYmes (CAZy) database ${ }^{(57)}$.

\section{Immunological and physiological measurements}

Methods used in measuring the impact of $\beta 2-1$ fructan supplementation on cytokines, immune cell population analysis and physiological markers have been previously described ${ }^{(9)}$. Lipopolysaccharide binding protein (LBP) was measured using the HK315 Human LBP ELISA kit (Hycult Biotech).

\section{Statistical analysis}

Associations between bacterial taxa and measured host parameters were tested by Pearson's or Spearman's (nonparametric) correlation analysis. Multiple $P$ values obtained from the large number of individual correlation tests were corrected for false discovery rate using a step-down Bonferroni correction. A corrected $P<0.050$ was considered significant. Unless indicated, analyses were performed using SAS Enterprise Guide version 5.1 (SAS Institute Inc.).

The effect of $\beta 2-1$ fructan induced community change over time on immune and other physiological parameters and was assessed by repeated measures ANOVA (type 3: treatment = repeated effect) with treatment, order and bacterial community profile as the main effects and with two interactions (treatment $\times$ bacterial community profile and treatment $\times$ order) included in the final model. Data were grouped by order to account for potential differences in the covariance structure of this parameter. Data were analysed as the difference (day 28-day 0) for the placebo and $\beta 2-1$ fructan supplementation phases. When a significant treatment effect was observed, data were visually inspected to ensure that changes were associated only with the $\beta 2-1$ supplementation phase (minimal changes occurred during the placebo supplementation phase). In cases when the data were not normally distributed, data were transformed using the Box-Cox procedure. When four or less observations were below the detection limit 
for a parameter, the data were analysed using the multiple Wilcoxon signed-rank test (Statistica version 7; StatSoft).

Ordinal and cluster analysis of 16S rRNA gene data, SEED and CAZy gene assignments were implemented using PC-ORD ${ }^{(58)}$ according to standard approaches for the analysis of ecological data $^{(59)}$. Matrices of phylotype abundance and distributions at the phylum, class, order, family, genus and phylotype for each subject ( $n$ 26) or SEED and CAZyme assignments $(n$ 4) at each sampling time were normalised and expressed as a percentage of the total bacterial community. Ordination analysis was implemented through PC-ORD with the analysis carried out in the autopilot mode (maximum 6-axis, 500 iterations). Optimal solutions were obtained using Non-metric Multidimensional Scaling (NMS) and a Euclidean distance measure. The primary sources of variation (changes in taxa in response to treatment) between the day 28 fructan faecal communities and all other faecal samples were identified using the joint-plot function in PC-ORD and an $r^{2}$ cutoff $=0 \cdot 250$. As NMS axis are not always arranged in terms of order of effect, ordinations were also carried out using principal coordinates analysis (PCoA; Euclidean distance measure) to confirm that the primary sources of variation were oriented on their appropriate axis of the NMS plots. Group testing was carried out using the multi-response permutation procedure ${ }^{(60)}$ using a Euclidean distance measure and implemented in PC-ORD. Cluster analysis was also implemented using PC-ORD using a Euclidean distance measure and a group average linkage method.

\section{Results}

Subject characteristics have been previously published $^{(9)}$; details concerning participant demographics ( $n$ 26) are shown in Table 1.

\section{Inulin utilisation within the cultivable faecal community}

In order to gain a more complete perspective on the species capable of utilising $\beta 2-1$ fructan, faeces collected at the termination of each treatment phase (day 28) were subjected to anaerobic cultivation using a modified L-10 anaerobic medium containing inulin as the sole carbohydrate source. In total, eighty-seven species encompassing thirty genera and four phyla were isolated (Fig. 2; online Supplementary Table S1). Most isolates were closely related to previously characterised species, although several are likely to be new taxa based on their 16S rRNA gene sequence divergence with existing species ( $>3 \%$ ). Isolates within the genus Bifidobacterium were the most common ( $32 \%$ of total), followed by Bacteroides (15\%), Ruminococcus (9\%), Enterococcus (9\%) and Blautia (6\%). The most abundant cultivated species was Bifidobacterium longum (13\% of total isolates). $\beta 2-1$ fructan supplementation had only marginal effects on the abundance and composition of species isolated across all thirty subjects (Fig. 2).

\section{Terminal restriction fragment length polymorphism community analysis}

Ordinal analysis (PCA) of T-RFLP profiles generated from day 28 faeces of subjects fed $\beta 2-1$ fructan were compared with faecal
Table 1. Subject demographic data at study baseline ( $n$ 26) (Mean values and standard deviations and $95 \%$ confidence intervals)

\begin{tabular}{|c|c|}
\hline Characteristics & $n$ \\
\hline \multicolumn{2}{|l|}{ Sex } \\
\hline Male & 11 \\
\hline Female & 15 \\
\hline \multicolumn{2}{|l|}{ Age (years) } \\
\hline Mean & $28 \cdot 2$ \\
\hline SD & $5 \cdot 1$ \\
\hline $95 \% \mathrm{Cl}$ & $25 \cdot 0,30 \cdot 5$ \\
\hline \multicolumn{2}{|l|}{ Height (m) } \\
\hline Mean & $1 \cdot 70$ \\
\hline $\mathrm{SD}$ & 0.10 \\
\hline $95 \% \mathrm{Cl}$ & $1.63,1.78$ \\
\hline \multicolumn{2}{|l|}{ Weight $(\mathrm{kg})$} \\
\hline Mean & 70.9 \\
\hline $\mathrm{SD}$ & 12.5 \\
\hline $95 \% \mathrm{Cl}$ & $59 \cdot 1,79 \cdot 3$ \\
\hline \multicolumn{2}{|l|}{$\mathrm{BMI}\left(\mathrm{kg} / \mathrm{m}^{2}\right)$} \\
\hline Mean & 24.5 \\
\hline SD & 3.0 \\
\hline $95 \% \mathrm{Cl}$ & $22 \cdot 6,26 \cdot 6$ \\
\hline \multicolumn{2}{|c|}{ Systolic blood pressure $(\mathrm{mmHg})$} \\
\hline Mean & $121 \cdot 3$ \\
\hline SD & $12 \cdot 0$ \\
\hline $95 \% \mathrm{Cl}$ & $113.5,131.3$ \\
\hline \multicolumn{2}{|c|}{ Diastolic blood pressure $(\mathrm{mmHg})$} \\
\hline Mean & $78 \cdot 1$ \\
\hline SD & 8.5 \\
\hline $95 \% \mathrm{Cl}$ & $69 \cdot 5,83 \cdot 3$ \\
\hline
\end{tabular}

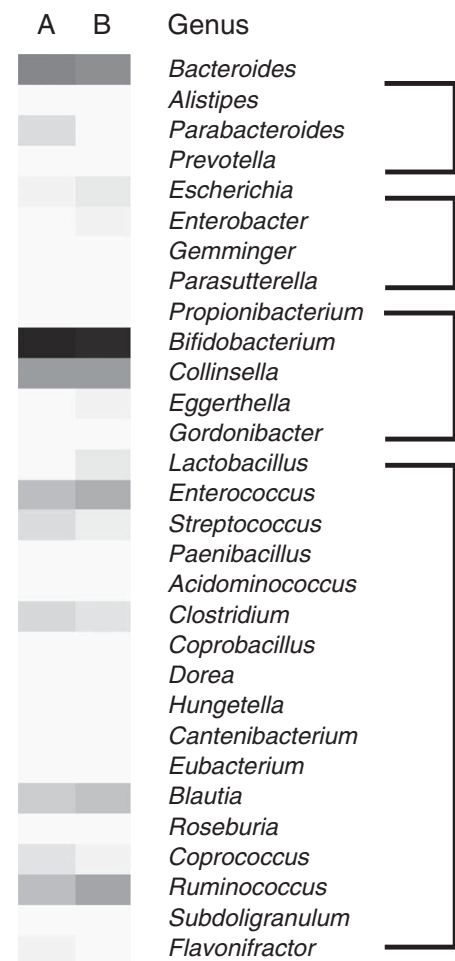

Phylum

Bacteroidetes

Proteobacteria

Actinobacteria

Firmicutes

Fig. 2. Heat plot showing the distribution of isolates at the genus and phylum level from the faeces of subjects fed the placebo $(A)$ or $\beta 2-1$ fructan supplement $(B)$. Information on the specific species isolated is available in online Supplementary Table S1.

community profiles from all subjects not receiving this supplement (placebo days 0 and 28, and fructan day 0). A difference in community structure was observed ( $P=0 \cdot 022$; Fig. 3(a)). 
(a)

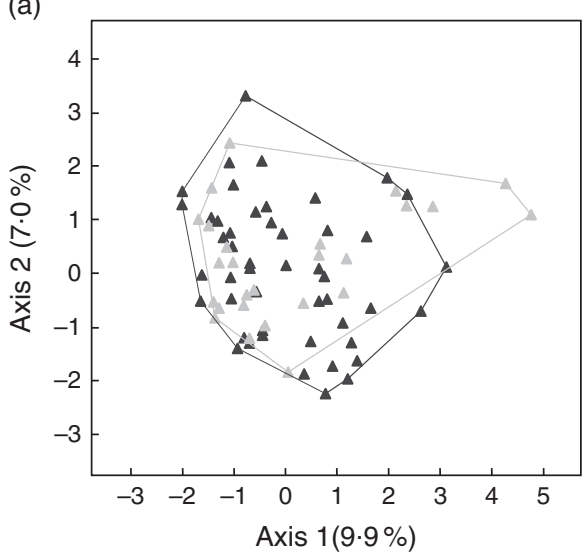

(c)

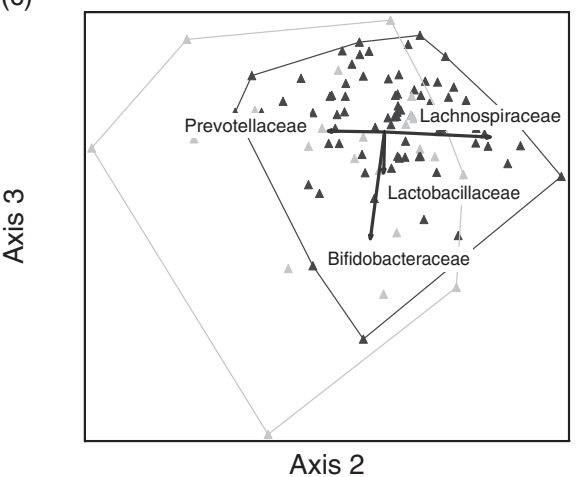

(b)

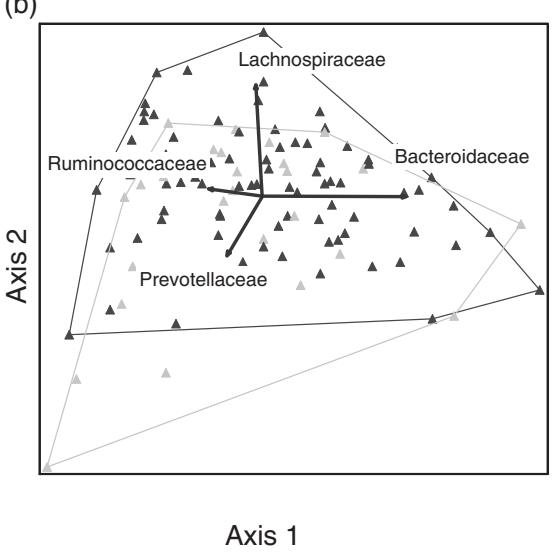

(d)

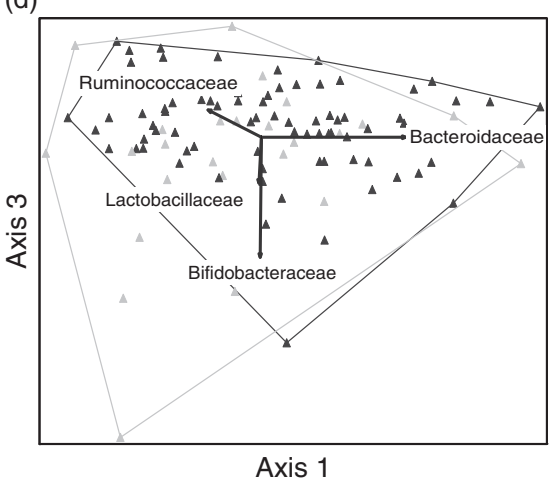

Fig. 3. Ordinal plots comparing faecal community structure between the day 28 samples from subjects fed $\beta 2-1$ fructan with the controls (days 0 and 28 placebo, day 0 $\beta 2-1$ fructan). Faecal communities from subjects fed $\beta 2-1$ fructan (day 28) are indicated by black triangles and controls by grey triangles. (a) Principal component analysis plot comparing faecal terminal restriction fragment length polymorphism profiles. Groups were different $(P=0.022)$. (b-d) Non-metric Multidimensional Scaling plots comparing faecal community structure at the family taxonomic level over the three primary axes. Lineages primarily responsible for variation between communities $\left(r^{2}>0.250\right)$ are indicated by joint plots. The length of the arrows indicates the relative strength of each factor and the direction of the arrow the axis of orientation. Corresponding correlation coefficients for each factor are available in Table 3.

\section{Sequence-based analyses of faecal communities}

In total, 387 phylotypes were identified in faecal communities from twenty-six subjects (online Supplementary Table S2 and S3). These aligned into eight phyla, although four (Firmicutes $>$ Bacteroidetes $>$ Actinobacteria $>$ Proteobacteria) encompassed the majority of faecal community diversity. Faecal community composition was unique to each individual and this did not change with treatment (online Supplementary Fig. S1). Previously, we determined levels of bifidobacteria in the faecal samples from each subject by quantitative PCR (qPCR) over the entire trial ${ }^{(9)}$. In order to corroborate the 16S rRNA gene-based analysis, we compared qPCR results with those derived from the sequence data (Fig. 4). The 16S rRNA gene sequence-based analysis over-estimated relative bifidobacteria content by approximately 2-fold, although the increased 16S rRNA gene content with $\beta 2-1$ fructan supplementation was consistent with previously reported qPCR analysis ${ }^{(9)}$.

\section{Faecal community change in response to $\beta 2-1$ fructans}

Faecal communities from subjects fed $\beta 2-1$ fructan (day 28) were less species rich than the corresponding day 0 and placebo phase samples (Table 2). Changes in faecal community structure in response to supplement (day $28 \beta 2-1$ fructan $v$. all other samples) were initially examined using ordinal analysis (NMS) and group testing, an approach commonly used in the analysis of ecological data ${ }^{(59)}$. Comparisons at the phylum, class, order and family levels are summarised in Table 3, and the ordinal analysis at the family level is presented in Fig. 3(b)-(d). No reasonable ordinal solution was found at the phylotype level. Differences between the day $28 \beta 2-1$ fructan faecal communities and all other communities were only observed at the genus level $(P=0 \cdot 026)$. However, this result must be viewed with caution as the ordinal solution was not only of lower quality (stress $=0 \cdot 160$ ), but substantial information was also lost due to the exclusion of phylotypes not assigned to specific genera.

In order to more clearly understand the impact of $\beta 2-1$ fructan supplementation on faecal community change, we first identified the taxa contributing the largest portion of community variation at each level of the taxonomic hierarchy between the day $28 \beta 2-1$ fructan supplemented faecal community and all other faecal samples (Table 3, Fig. 3). At phylum, class, order and family levels two lineages represented the largest sources of variation between the day $28 \beta 2-1$ fructan phase and all other samples, and these 
were Bacteroidetes (Bacteroidia, Bacteroidales, Bacteroidaceae) and Firmicutes (Clostridia, Clostridiales, Ruminococcaceae). Respective abundance of bacteria within these two phyla were

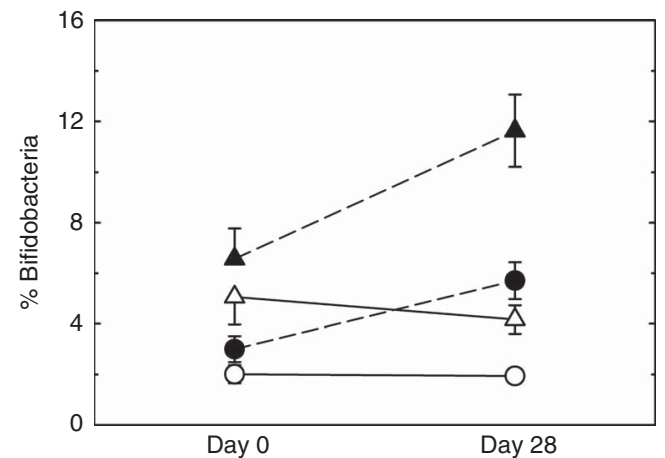

Fig. 4. Abundance of bifidobacteria (\% of total community) in faeces of subjects fed $\beta 2-1$ fructan (days 0 and 28) or maltose (days 0 and 28) as determined by quantitative PCR (qPCR) ${ }^{(9)}$ and by analyses of $16 \mathrm{~S}$ rRNA gene libraries. $-0-$, qPCR control; $-0-$, qPCR $\beta 2-1$ fructan; $\square-$, 16S rRNA control; $-\mathbf{\Lambda}-$, , $16 \mathrm{~S}$ rRNA $\beta 2-1$ fructan.

Table 2. Estimates of species richness (Abundance-based Coverage Estimator $(A C E)^{(61)}$ ) in the faecal communities of subjects (twenty-six) supplemented with $\beta 2-1$ fructan (day 28 ) and controls (days 0 and 28 placebo, day $0 \beta 2-1$ fructan)*

(Mean values with their standard errors)

\begin{tabular}{lcc}
\hline & \multicolumn{2}{c}{ ACE } \\
\cline { 2 - 3 } Sample & Mean & SEM \\
\hline Placebo day 0 & $53 \cdot 3$ & $2 \cdot 3$ \\
Placebo day 28 & $53 \cdot 8$ & $2 \cdot 4$ \\
$\beta 2-1$ fructan day 0 & $52 \cdot 4$ & $2 \cdot 4$ \\
$\beta 2-1$ fructan day 28 & $45 \cdot 3$ & $2 \cdot 3$ \\
\hline
\end{tabular}

* Subjects fed $\beta 2-1$ fructan are less rich than the control communities $(P=0.002)$. also negatively correlated with each other (online Supplementary Fig. S2(A)). Although additional lineages also contributed to variation within the community (Actinobacteria, Bacilli), these were not dominant effects as their corresponding biplots aligned parallel with the second or third axis of both NMS (Table 3) and PCoA ordinal plots (results not shown).

At the family level, change in the abundance of the Bacteroidaceae was the primary source of variation between the day 28 32-1 fructan communities and all other samples (Table 3, Fig. 3). Dividing the cohort on this basis revealed two primary patterns in the community response to $\beta 2-1$ fructan supplementation. In nine subjects, the abundance of phylotypes aligning with Bacteroidetes increased, whereas those aligning within Lachnospiraceae and Ruminococcaceae (primarily the genus Faecalibacterium) decreased (Fig. 5). In the remaining subjects $(17 / 26), \quad \beta 2-1$ fructan supplementation tended to decrease the abundance of phylotypes aligning in the family Bacteroidaceae while phylotypes aligning with Lachnospiraceae, Ruminococcaceae (genus Faecalibacterium) and Lactobacillaceae increased (Fig. 5). The subjects falling into the first pattern could be further separated into those where the increase in Bacteroidaceae fell primarily within the genus Bacteroides (5/9; Fig. 5(a)) and those where the increase fell within the family Prevotellaceae (4/9; Fig. 5(b)). Relative bifidobacteria levels increased in response to $\beta 2-1$ fructan supplementation and this was particularly noted in those subjects where Bacteroidetes trended downward (Fig. 5(c)). Communities in those subjects (17/26) who responded to $\beta 2-1$ fructan by decreasing the content of Bacteroidetes were different from those who responded by increasing abundance within this lineage $(9 / 26 ; P=0.027)$. Subjects who responded to $\beta 2-1$ fructan by increasing faecal Bacteroidetes content are indicated in online Supplementary Table S3.

Table 3. Identification of taxa responsible for variation in the faecal communities in response to $\beta 2-1$ fructan supplementation*

\begin{tabular}{|c|c|c|c|c|c|c|c|}
\hline Taxa & $\%$ Coverage & Ordinal solution (NMS) & Stress & Lineage & $r^{2}+$ & Ordinal axis & MRPP‡ \\
\hline \multirow[t]{2}{*}{ Phylum } & $99 \cdot 6+0 \cdot 7$ & $2 \mathrm{D}$ & 0.085 & Firmicutes & 0.926 & 1 & NS \\
\hline & & & & Bacteroidetes & 0.909 & 1 & \\
\hline \multirow[t]{4}{*}{ Class } & $99.6+0.7$ & $2 \mathrm{D}$ & 0.041 & Clostridia & 0.915 & 1 & NS \\
\hline & & & & Bacteroidia & 0.907 & 1 & \\
\hline & & & & Actinobacteria & 0.877 & 2 & \\
\hline & & & & Bacilli & 0.349 & 2 & \\
\hline \multirow[t]{3}{*}{ Order } & $98 \cdot 9+2 \cdot 0$ & $2 \mathrm{D}$ & 0.060 & Clostridiales & 0.927 & 1 & NS \\
\hline & & & & Bacteroidales & 0.860 & 1 & \\
\hline & & & & Bifidobacteriales & 0.877 & 2 & \\
\hline \multirow[t]{6}{*}{ Family } & $95 \cdot 8+6 \cdot 9$ & $3 \mathrm{D}$ & 0.070 & Bacteroidaceae & 0.974 & 1 & NS \\
\hline & & & & Ruminococcaceae & 0.364 & 1 & \\
\hline & & & & Lachnospiraceae & 0.749 & 2 & \\
\hline & & & & Prevotellaceae & 0.476 & 2 & \\
\hline & & & & Bifidobacteriaceae & 0.726 & 3 & \\
\hline & & & & Lactobacillaceae & 0.278 & 3 & \\
\hline \multirow[t]{5}{*}{ Genus } & $88 \cdot 0+11 \cdot 4$ & $2 \mathrm{D}$ & 0.160 & Bacteroides & 0.896 & 1 & 0.026 \\
\hline & & & & Prevotella & 0.389 & 1 & \\
\hline & & & & Bifidobacterium & 0.440 & 2 & \\
\hline & & & & Faecalibacterium & 0.280 & 2 & \\
\hline & & & & Lactobacillus & 0.276 & 2 & \\
\hline Phylotype & 100 & No reasonable solution & & & & & \\
\hline
\end{tabular}

MRPP, multi-response permutation procedure; NMS, Non-metric Multidimensional Scaling.

* Comparisons are between day 28 of the $\beta 2-1$ fructan phase with the corresponding day 0 , days 0 and 28 of the placebo phase ( $n$ 26). Ordinal analysis (NMS) at the family level is shown in Fig. 3.

† Correlation coefficients $\left(r^{2}\right)$ for the primary lineages contributing to community variation (joint plots) on NMS ordinal plots. Only those $r^{2}>0.250$ are shown.

$\ddagger$ Group testing (day 28 fructan community $v$. all others) as determined using MRPP using a Euclidean distance measure. NS: $P>0.050$. 
(a)

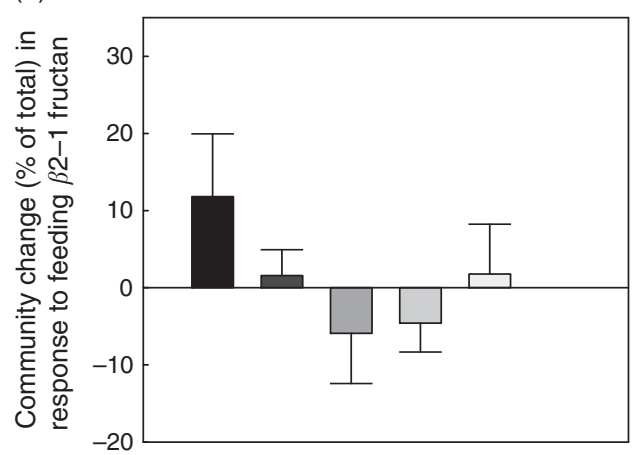

(b)

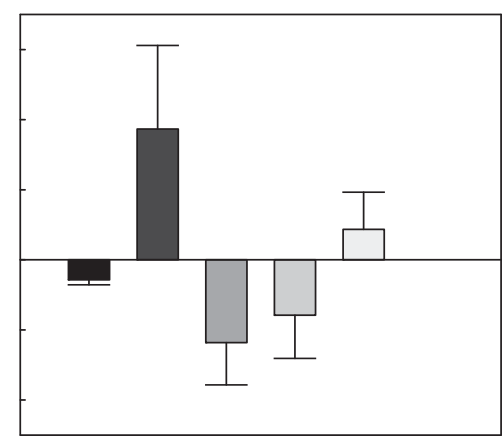

(c)

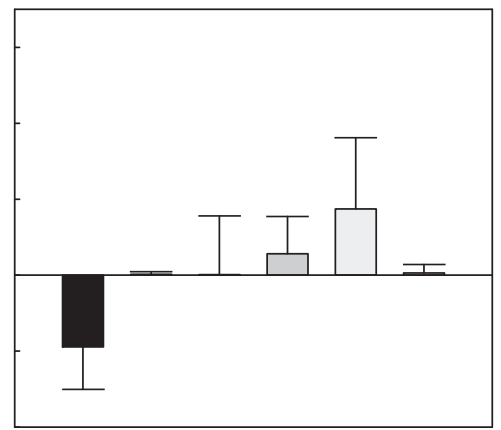

Fig. 5. Change in relative content ( $\%$ of total $16 \mathrm{~S}$ rRNA genes) of family lineages contributing the largest portion of variation. Comparisons are between $\beta 2-1$ fructan (day 28) faecal communities and control communities (mean of days 0 and 28 of the placebo phase and day 0 of the $\beta 2-1$ fructan phase). Subjects ( $n 9$ ) where Bacteroidetes levels trend upwards in response to $\beta 2-1$ fructan: (a) Bacteroidaceae or (b) Prevotellaceae. Subjects ( $n$ 17) where Bacteroides levels tend downwards in response to $\beta 2-1$ fructan (c). Values are means and standard deviations represented by vertical bars. $\square$, Bacteroidaceae; $\square$, Prevotellaceae; $\square$, Lachnospiraceae; $\square$, Ruminococcaceae; $\square$, Bifidobacteriaceae; $\square$, Lactobacillaceae.

Table 4. Correlations between the faecal bacterial community and various host parameters

\begin{tabular}{lrcc}
\hline Correlations $^{*}$ & $r$ & $r^{2} \dagger$ & $P \ddagger$ \\
\hline Bacteroidetes $v$. Firmicutes & -0.838 & 0.702 & 0.003 \\
Bacteroidaceae $v$. Ruminococcaceae & -0.575 & 0.331 & 0.035 \\
Bacteroidetes $v$. propionic acid & 0.658 & 0.433 & 0.011 \\
Firmicutes $v$. propionic acid & -0.496 & 0.246 & 0.011 \\
Ruminococcaceae $v$. propionic acid & -0.567 & 0.322 & 0.043 \\
Bacteroidaceae $v$. propionic acid & 0.498 & 0.248 & 0.043 \\
\hline
\end{tabular}

* Pearson's correlation.

† Only those having $r^{2} \geq 0.250$ have been reported.

$\ddagger$ Corrected for false discovery rate (FDR) using a step-down Bonferroni.

\section{Community change and its impact on host immune and physiological parameters}

A correlation analysis was undertaken to identify potential relationships between intestinal community lineages and previously reported immunologic parameters (sCD40L, granulocyte colonystimulating factor (G-CSF), granulocyte-macrophage colonystimulating factor (GM-CSF), interferon- $\gamma$, IL-1 $\beta$, IL-1Ra, IL-4, IL-6, IL-8, IL-10, IL12p70, IP-10, TNF- $\alpha$, active and total transforming growth factor- $\beta 1$ concentrations, percentages of $\mathrm{CD}_{28} 2^{+}$and $\mathrm{CD} 284^{+} \mathrm{mDC}$ and granulocytes), circulating physiological markers (C-reactive protein, blood urea N, serum lipopolysaccharide (LPS) and LBP), faecal SCFA, and self-reported health complaints. Few significant associations were detected, although positive correlations between the content of faecal Bacteroidetes and faecal propionate were observed (online Supplementary Fig. S2 (B); Table 4). Subjects having lower faecal Bacteroidetes content exhibited higher serum LPS and LBP concentrations independent of treatment (Table 5), whereas those with increased faecal Bacteroidetes produced more faecal caproic acid independent of treatment (Table 5).

\section{Metagenomic analysis}

In order to gain insight into the effect of $\beta 2-1$ fructan supplementation on the faecal community metagenome, we carried out high-throughput sequencing and assembly of shotgun clones from faecal communities of four subjects (Subjects 1, 6, 24 and 25) over the entire duration of the trial. These four subjects were representative of the primary changes identified in the cohort in response to $\beta 2-1$ fructan supplementation (online Supplementary Fig. S3). Gene assignments into the major SEED categories were similar among subjects, despite the differences in faecal community composition (online Supplementary Fig. S4 and Table S5). Similar to previous examinations of human faecal communities, metagenomes were enriched in genes involved in the metabolism of carbohydrates and depleted in those encoding motility and chemotaxis ${ }^{(62)}$. Cluster analysis of SEED gene assignments for each faecal sample is shown in Fig. 6(a). Group testing found no relationship between clusters and treatment $(P=0.816)$, although the metagenome of subject 6 was marginally different from the other subjects $(P=0 \cdot 044)$. Contiguous DNA sequences were also compared against the CAZy database to examine potential changes in glycosyl-hydrolase (GH) capabilities of these communities (online Supplementary Table S6). $\mathrm{GH}$ profiles clustered by subject and were different $(P<0 \cdot 008$; Fig. 6(b)). However, GH profiles in the day $28 \beta 2-1$ fructan phase were not different from the other samples within each subject, suggesting that differences in clustering within individual subjects likely reflect temporal variability associated with each subject's faecal community.

\section{Discussion}

Supplementing humans with $\beta 2-1$ fructan increases the abundance of faecal bifidobacteria ${ }^{(7-9,11)}$ and this has generally been attributed to the selective ability of this genus to utilise this substrate $^{(7)}$. We observed no increase in the abundance of cultivable bifidobacteria in response to $\beta 2-1$ fructan feeding, although our analysis was undertaken using a non-selective medium rather than one designed for enumeration of bifidobacteria. Although species within this genus were the most abundant $\beta 2-1$ fructan utilizers, we also isolated a wide range of additional species sharing this ability, some of which are previously known to metabolise $\beta 2-1$ fructans ${ }^{(34-36)}$. The phenotype also appears very common in species throughout the Actinobacteria, Firmicutes and Proteobacteria, and together 
Table 5. Relationships between the faecal bacterial community and various host parameters (Mean values with their standard errors (according to community profile))

\begin{tabular}{|c|c|c|c|c|c|c|c|c|c|c|c|}
\hline \multirow[b]{3}{*}{ Measure } & \multirow[b]{3}{*}{ Community profile } & \multicolumn{4}{|c|}{ Placebo } & \multicolumn{4}{|c|}{$\beta 2-1$ Fructan } & & \\
\hline & & \multicolumn{2}{|c|}{ Day 0} & \multicolumn{2}{|c|}{ Day 28} & \multicolumn{2}{|c|}{ Day 0} & \multicolumn{2}{|c|}{ Day 28} & \multicolumn{2}{|c|}{$P$} \\
\hline & & Mean & SE & Mean & SE & Mean & SE & Mean & SE & Treatmentł & Profile† \\
\hline \multirow[t]{2}{*}{ LPS (EU/ml) } & Increased Bacteroidetes & 0.33 & 0.10 & 0.51 & 0.18 & 0.34 & 0.05 & 0.51 & 0.14 & NS & 0.039 \\
\hline & Decreased Bacteroidetes & 0.60 & 0.14 & 0.51 & 0.11 & 0.61 & 0.11 & 0.71 & 0.16 & & \\
\hline \multirow[t]{2}{*}{ LBP $(\mu \mathrm{g} / \mathrm{ml})$} & Increased Bacteroidetes & $10 \cdot 7$ & 1.3 & $15 \cdot 7$ & 3.5 & $11 \cdot 2$ & 1.8 & 13.4 & $2 \cdot 1$ & NS & 0.026 \\
\hline & Decreased Bacteroidetes & $15 \cdot 5$ & 1.8 & $16 \cdot 8$ & $2 \cdot 3$ & $16 \cdot 4$ & $2 \cdot 3$ & $16 \cdot 2$ & $2 \cdot 1$ & & \\
\hline \multirow[t]{2}{*}{ Caproic acid } & Increased Bacteroidetes & 0.69 & 0.35 & 0.76 & 0.31 & 0.93 & 0.33 & 0.29 & 0.13 & NS & 0.006 \\
\hline & Decreased Bacteroidetes & 0.49 & 0.18 & 0.45 & 0.18 & 0.42 & 0.16 & 0.49 & 0.18 & & \\
\hline
\end{tabular}

LPS, lipopolysaccharide; LBP, lipopolysaccharide binding protein.

* Increased Bacteroidetes: $n$ 9, decreased Bacteroidetes: $n 17$.

$\dagger$ Differences tested using raw data.

‡ Differences between treatment phases tested using normalised values ((days 28-day0)/day 0)

(a)

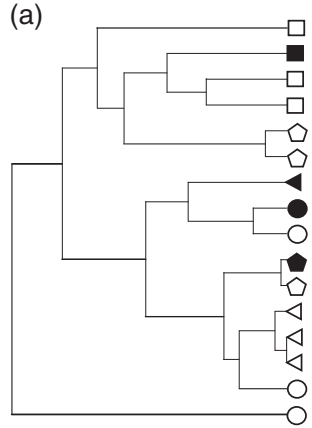

(b)

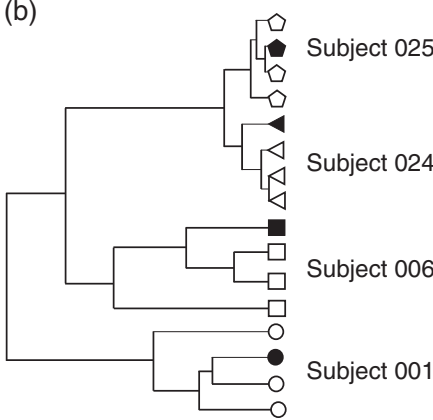

Fig. 6. Cluster diagrams showing the relationships among faecal metagenomes (a) and CAZyme gene assignments (b) from Subjects 1, 6, 24 and 25. $\square, \boldsymbol{\Delta}, \boldsymbol{a}$ and , Day $28 \beta 2-1$ fructan faecal communities; $\square, \triangle, \bigcirc$ and $\square$, corresponding controls (day $0 \beta 2-1$ fructan, days 0 and 28 placebo). Subjects and their corresponding symbols are identified on the right of the figure. SEED assignments are available in online Supplementary Table S5. CAZyme assignments are available in online Supplementary Table S6. Clustering was carried out using a Euclidean distance measure and a group average linkage method.

challenges the perspective regarding the selective nature of this substrate.

We examined the effect of $\beta 2-1$ fructan on faecal community structure and composition in healthy subjects using two molecular-based approaches. In the first case, analysis of T-RFLP profiles showed that consuming supplemental $\beta 2-1$ fructan altered faecal community structure. Our second approach based on the analysis of community 16S rRNA genes failed to detect any differences between treatments, although we did note that $\beta 2-1$ fructan reduced faecal community phylotype richness. Typically, 16S rRNA gene data are treated as quantitative data. However, there is a need to apply a degree of caution when interpreting results from this type of analysis as the method suffers limitations ${ }^{(63)}$, and even under optimised conditions community structures are distorted to varying degrees due to differences in 16S rRNA gene copy numbers between different $\operatorname{taxa}^{(64)}$. In order to understand the global impact of $\beta 2-1$ fructan supplementation, we first identified the bacterial lineages contributing the most community variation in response to treatment. The single largest source of variation lay within the Bacteroidetes. Based on changes in the relative abundance within this lineage, we identified two primary responses to feeding $\beta 2-1$ fructan. Interestingly, bifidobacteria were found to be one of the lesser sources of community variation, unexpected given that change within this taxon is widely accepted as a marker for the prebiotic effect ${ }^{(7,37)}$. Overall, the trends towards increased bifidobacteria and Faecalibacterium, and decreased Bacteroidetes observed in the majority of subjects are consistent with more recent work carried out in human beings fed $\beta 2-1$ fructans ${ }^{(12,19,65)}$. In contrast, the response in those subjects where faecal Bacteroidetes increased was similar to the response observed in female rats fed $\beta 2-1$ fructan $^{(66)}$.

Diet mediated change in intestinal communities is generally viewed strictly in terms of carbohydrate availability, and the contribution of gut $\mathrm{N}$ as a factor affecting community structure is often overlooked ${ }^{(50,67,68)}$. In rats, diet mediated changes to bacterial community structure can also be driven by changes in the availability of $\mathrm{N}$ (ammonia $v$. peptidyl $\mathrm{N}$ ) used to support bacterial growth. For example, rat caecal and faecal communities have higher Bacteroidetes content when ammonia is the primary $\mathrm{N}$ source used to support growth, whereas increasing peptidyl $\mathrm{N}$ availability drives Bacteroidetes content down and Lachnospiraceae content up ${ }^{(50,66,68)}$. These diet mediated responses observed in rats share similarities with the two patterns of faecal community change that we observed in humans ingesting $\beta 2-1$ fructan. This raises the question of whether the bifidogenic effect, as well as the other associated community changes, might partially reflect changes in fixed $\mathrm{N}$ availability in the large intestine as opposed to a selective growth stimulation resulting from the fermentation of $\beta 2-1$ fructan. Interestingly, the response of Faecalibacterium mirrored that of bacteria within the family Lachnospiraceae, which suggests a dependence on peptidyl $\mathrm{N}$ as the preferred $\mathrm{N}$ source in this species.

An important limitation with studies examining the effect of diet on human intestinal communities is the dependence on faecal samples. We have little to no information on how different fermentable substrates affect the bacterial communities in the human proximal colon. In rats, the fermentation of $\beta 2-1$ fructan is both rapid and occurs primarily in the caecum ${ }^{(28)}$. In human beings, fermentation likely occurs in the proximal (ascending) colon based on results from breath $\mathrm{H}$ analysis using 
inulin $^{(69)}$, and the fact that this represents the most active site for fermentation $^{(70)}$. In rats, the intensity of the caecal fermentation of $\beta 2-1$ fructan increases mucin excretion, and reduces barrier integrity as indicated by increased translocation of enteric bacteria ${ }^{(71-74)}$. Similarly, we observed changes in various host parameters in subjects administered $\beta 2-1$ fructan consistent with a reduction in intestinal barrier function ${ }^{(9)}$. Moreover, mucin excretion in response to $\beta 2-1$ fructan has been previously observed in human subjects ${ }^{(30)}$, although not all studies have shown this ${ }^{(32)}$. However when considered together, it is not unreasonable to suggest that the response in humans to $\beta 2-1$ fructan shares many similarities with the well documented response in rats.

Although increases in bifidobacteria are consistent with the widely held view that the enrichment is attributable to their selective ability to metabolise $\beta 2-1$ fructans $^{(7)}$, we suggest that the community changes observed here might be better explained on the basis of mucin foraging in the descending colon. First, the ability to ferment mucin, the O-side chain, or the carbohydrate constituents by various intestinal bacteria ${ }^{(75)}$, including bifidobacteria ${ }^{(76,77)}$, Bacteroides ${ }^{(78)}$ and Faecalibacterium $^{(79)}$ is well established. Second, mucin being a glycoprotein also represents a source of peptidyl $\mathrm{N}$ and its presence in the descending colon due to sloughing would be consistent with the dominant pattern of change observed across the cohort consuming $\beta 2-1$ fructans. In fact, the two primary patterns of bacterial community response observed across our cohort might ultimately reflect differences in inter-individual variation in the extent of mucin sloughing in response to feeding this rapidly fermented substrate. A distal colonic fermentation affected by mucin availability not only resolves the dilemma regarding the selective effects of a substrate which is metabolised by a very wide range of intestinal bacteria, but also why a bifidogenic response occurs following the feeding of other rapidly fermented oligosaccharides ${ }^{(37-40)}$, as well as the variation in the extent of the bifidogenic effect observed amongst individuals ${ }^{(37)}$.

Although $\beta 2-1$ fructan supplementation altered the faecal bacterial community of these subjects, we observed few relationships between community changes and host parameters. However, a relationship between the abundance of Bacteroidetes and concentrations of faecal propionate was observed, consistent with a previous study in humans ${ }^{(80)}$. We also observed a relationship between subjects having lower faecal Bacteroidetes content and increased serum LPS and LBP concentrations, which occurred independently of treatment. LBP binds LPS and other bacterial wall components forming a complex that interacts with CD14 mediating the activation of peripheral blood monocytes via TLR $4^{(81)}$. Normally LBP is present in serum at concentrations ranging from $5-15 \mu \mathrm{g} / \mathrm{ml}$; these subjects being at the higher end of the normal range. Although these findings may be coincidental, they do suggest a difference in gut barrier functionality between the two response groups within the cohort.

In conclusion, we have examined faecal community change in a cohort of healthy human subjects supplemented with $\beta 2-1$ fructan. Although we observed two primary patterns of response in the faecal communities of these subjects, these changes were nonetheless consistent with previous work in both rats and human subjects ${ }^{(12,19,66)}$. Changes in faecal bacterial community structure may be affected by mucin availability with the two patterns of change reflective of differences in the availability of peptidyl $\mathrm{N}$ in the distal colon. Overall, the extent of change in the faecal bacterial communities of subjects was quite modest, and this is supported by the metagenome and CAZyme profiles where no discernable treatment effect was observed. The prebiotic health claim is based on establishing a link between a change in the intestinal community and a repeatable/measurable health effect ${ }^{(82)}$, although a correlation could also be considered as supporting evidence ${ }^{(83)}$. Although it has been argued that shifting fermentation distally to provide SCFA to the distal colon through mucin fermentation ${ }^{(84)}$, or that reducing concentrations of secondary metabolites from fermentation of proteins is beneficial ${ }^{(9,33)}$, these potential benefits should be considered against the impact of the $\beta 2-1$ fructan fermentation on reducing intestinal barrier function ${ }^{(9,30,74)}$. Moreover, similar effects would be shared with other rapidly fermented carbohydrates. Although it remains possible that a health effect may be discovered in studies examining larger cohorts, our findings ${ }^{(9)}$ as well as those of others ${ }^{(8,10,11,30)}$ do not support the premise that dietary $\beta 2-1$ fructans confer a demonstrable health benefit in healthy adult human subjects.

\section{Acknowledgements}

The authors wish to thank Fernando Matias (Health Canada), Mark Ritchie and Jenny Gusse (AAFC Kentville and Lethbridge respectively) for their excellent technical assistance.

This trial was undertaken with financial support from Agriculture and Agri-Food Canada (RPBI no. 1501, M. K., G. D. I., L. J. Y.), Health Canada (S. P. J. B.), Alberta Innovates Bio Solutions (G. D. I., L. J. Y., M. K., S. P. J. B., J. M. G.-J.), and the Advanced Food and Materials Network (M. K.) and the National Science and Engineering Research Council (J. M. G.-J.).

Researchers in this group were involved in the original conception of the project (J. M. G.-J., D. D. R., P. B., G. D. I., L. J. Y., S. P. J. B., M. K.). D. D. R. and J. G. organised and supervised the clinical trial. G. D. I. was responsible for the T-RFLP analysis and sequencing of the 16S rRNA gene of cultivated bacteria, L. J. Y. carried out isolation of bacteria and the cultivation-based analysis, N. P. and M. K. were responsible for the sequencebased analyses. S. T. C. and S. P. J. B. undertook and provided the statistical analyses. S. T. C., J. M. G.-J. and M. K. prepared the first draft of the manuscript. All additional authors (S. P. J. B., D. D. R., P. B., G. D. I., J. G., L. J. Y., N. P. and L. B. S.) contributed to the final content of the manuscript.

All authors declare that there are no conflicts of interest.

\section{Supplementary material}

For supplementary material/s referred to in this article, please visit https://doi.org/10.1017/S0007114517002318

\section{References}

1. Kau AL, Ahern PP, Griffin NW, et al. (2011) Human nutrition, the gut microbiome and the immune system. Nature 474, 327-336. 
2. Brownawell AM, Caers W, Gibson GR, et al. (2012) Prebiotics and the health benefits of fiber: current regulatory status, future research, and goals. J Nutr 142, 962-974.

3. Claus SP \& Swann JR (2013) Nutrimetabonomics: applications for nutritional sciences, with specific reference to gut microbial interactions. Annu Rev Food Sci Technol 4, 381-399.

4. Salerno F, Moser P, Maggi A, et al. (1994) Effects of long-term administration of low-dose lactitol in patients with cirrhosis but without overt encephalopathy. J Hepatol 21, 1092-1096.

5. Morgan MY \& Hawley KE (1987) Lactitol vs. lactulose in the treatment of acute hepatic encephalopathy in cirrhotic patients: a double-blind, randomized trial. Hepatology $\mathbf{7}$, $1278-1284$.

6. Weber FL Jr (1997) Effects of lactulose on nitrogen metabolism. Scand J Gastroenterol 32, Suppl. 222, 83-87.

7. Gibson GR \& Roberfroid MB (1995) Dietary modulation of the human colonic microbiota: introducing the concept of prebiotics. J Nutr 125, 1401-1412.

8. Lomax AR, Cheung LV, Tuohy KM, et al. (2012) $\beta 2-1$ Fructans have a bifidogenic effect in healthy middle-aged human subjects but do not alter immune responses examined in the absence of an in vivo immune challenge: results from a randomised controlled trial. Br J Nutr 108, 1818-1828.

9. Clarke ST, Green-Johnson JM, Brooks SPJ, et al. (2016) $\beta 2-1$ Fructan supplementation alters host immune responses in a manner consistent with increased exposure to microbial components: results from a double-blinded, randomised, cross-over study in healthy adults. Br Nutr 115, 1748-1759.

10. Slavin J \& Feirtag J (2011) Chicory inulin does not increase stool weight or speed up intestinal transit time in healthy male subjects. Food Funct 2, 72-77.

11. Bouhnik Y, Flourié B, Riottot M, et al. (1996) Effects of fructooligosaccharides ingestion on fecal bifidobacteria and selected metabolic indexes of colon carcinogenesis in healthy humans. Nutr Cancer 26, 21-29.

12. Dewulf EM, Cani PD, Claus SP, et al. (2012) Insight into the prebiotic concept: lessons from an exploratory, double blind intervention study with inulin-type fructans in obese women. Gut 63, 1112-1121.

13. Gibson PR \& Shepherd SJ (2010) Evidence-based dietary management of functional gastrointestinal symptoms: The FODMAP approach. J Gastroenterol Hepatol 25, 252-258.

14. Halmos EP, Power VA, Shepherd SJ, et al. (2014) A diet low in FODMAPs reduces symptoms of irritable bowel syndrome. Gastroenterology 146, 67-75.

15. Singh RS \& Singh RP (2010) Production of fructooligosaccharides from inulin by endoinulinases and their prebiotic potential. Food Technol Biotechnol 48, 435.

16. Pollock CJ \& Cairns AJ (1991) Fructan metabolism in grasses and cereals. Ann Rev Plant Physiol 42, 77-101.

17. Coussement PA (1999) Inulin and oligofructose: safe intakes and legal status. J Nutr 129, 1412S-1417S.

18. Bouhnik Y, Raskine L, Simoneau G, et al. (2006) The capacity of short-chain fructooligosaccharides to stimulate faecal bifidobacteria: a dose-response relationship study in healthy humans. Nutr J 5, 8 .

19. Ramirez-Farias C, Slezak K, Fuller Z, et al. (2009) Effect of inulin on the human gut microbiota: stimulation of Bifidobacterium adolescentis and Faecalibacterium prausnitzii. Br J Nutr 101, 541-550.

20. Langkamp-Henken B, Rowe CC, Ford AL, et al. (2015) Bifidobacterium bifidum $\mathrm{R} 0071$ results from a greater proportion of healthy days and a lower percentage of academically stressed students reporting a day of cold/flu: a randomized, double-blind, placebo-controlled study. Br J Nutr 113, 426-434.
21. Hütt P, Shchepetova J, Loivukene K, et al. (2006) Antagonistic activity of probiotic lactobacilli and bifidobacteria against entero-and uropathogens. J Appl Microbiol 100, 1324-1332.

22. Fukuda S, Toh H, Taylor TD, et al. (2012) Acetate-producing bifidobacteria protect the host from enteropathogenic infection via carbohydrate transporters. Gut Microbes 3, 449-454.

23. Marteau P, Cuillerier E, Meance S, et al. (2002) Bifidobacterium animalis strain DN-173 010 shortens the colonic transit time in healthy women: a double-blind, randomized, controlled study. Aliment Pharmacol Ther 16, 587-593.

24. Lindsay JO, Whelan K, Stagg AJ, et al. (2006) Clinical, microbiological, and immunological effects of fructo-oligosaccharide in patients with Crohn's disease. Gut 55, 348-355.

25. Reddy BS, Hamid R \& Rao CV (1997) Effect of dietary oligofructose and inulin on colonic preneoplastic aberrant crypt foci inhibition. Carcinogenesis 18, 1371-1374.

26. Tsukahara T, Iwasaki Y, Nakayama K, et al. (2003) Stimulation of butyrate production in the large intestine of weaning piglets by dietary fructooligosaccharides and its influence on the histological variables of the large intestinal mucosa. J Nutr Sci Vitaminol 49, 414-421.

27. Davidson MH, Maki KC, Synecki C, et al. (1998) Effects of dietary inulin on serum lipids in men and women with hypercholesterolemia. Nutr Res 18, 503-517.

28. Campbell JM, Fahey GC \& Wolf BW (1997) Selected indigestible oligosaccharides affect large bowel mass, cecal and fecal short-chain fatty acids, $\mathrm{pH}$ and microflora in rats. J Nutr $\mathbf{1 2 7}$, 130-136.

29. Le Blay G, Michel C, Blottière HM, et al. (1999) Prolonged intake of fructo-oligosaccharides induces a short-term elevation of lactic acid-producing bacteria and a persistent increase in caecal butyrate in rats. J Nutr 129, 2231-2235.

30. Ten Bruggencate SJM, Bovee-Oudenhoven IMJ, LettinkWissink MLG, et al. (2006) Dietary fructooligosaccharides affect intestinal barrier function in healthy men. J Nutr $\mathbf{1 3 6}$, $70-74$.

31. Lomax AR, Cheung LV, Noakes PS, et al. (2015) Inulin-type $\beta 2-1$ fructans have some effect on the antibody response to seasonal influenza vaccination in healthy middle aged humans. Front Immunol 6, 490.

32. Scholtens PA, Alles MS, Willemsen LE, et al. (2006) Dietary fructo-oligosaccharides in healthy adults do not negatively affect faecal cytotoxicity: a randomised, double-blind, placebo-controlled crossover trial. Br J Nutr 95, 1143-1149.

33. Geboes KP, De Hertogh G, De Preter V, et al. (2006) The influence of inulin on the absorption of nitrogen and the production of metabolites of protein fermentation in the colon. Br J Nutr 96, 1078-1086.

34. Van der Meulen R, Makras L, Verbrugghe K, et al. (2006) In vitro kinetic analysis of oligofructose consumption by Bacteroides and Bifidobacterium spp. indicates different degradation mechanisms. Appl Environ Microbiol 72, 1006-1012.

35. Gourgue-Jeannot C, Kalmokoff ML, Kheradpir E, et al. (2006) Dietary fructooligosaccharides alter the cultivatable faecal population of rats but do not stimulate the growth of intestinal bifidobacteria. Can J Microbiol 52, 924-933.

36. Oyarzabal OA \& Conner DE (1995) In vitro fructooligosaccharide utilization and inhibition of Salmonella spp. by selected bacteria. Poult Sci 74, 1418-1425.

37. Macfarlane SMGT, Macfarlane GT \& Cummings JT (2006) Review article: prebiotics in the gastrointestinal tract. Aliment Pharmacol Ther 24, 701-714.

38. Depeint F, Tzortzis G, Vulevic J, et al. (2008) Prebiotic evaluation of a novel galactooligosaccharide mixture produced by the enzymatic activity of Bifidobacterium bifidum NCIMB 41171, in healthy humans: a randomized, double-blind, 
crossover, placebo-controlled intervention study. Am J Clin Nutr 87, 785-791.

39. Gostner A, Blaut M, Schäffer V, et al. (2006) Effect of isomalt consumption on faecal microflora and colonic metabolism in healthy volunteers. Br J Nutr 95, 40-50.

40. Finney M, Smullen J, Foster HA, et al. (2007) Effects of low doses of lactitol on faecal microflora, $\mathrm{pH}$, short chain fatty acids and gastrointestinal symptomology. Eur J Nutr $\mathbf{4 6}$, 307-314.

41. Dethlefsen L, Huse S, Sogin ML, et al. (2008) The pervasive effects of an antibiotic on the human gut microbiota, as revealed by deep 16S rRNA sequencing. PLoS Biol 6, e280.

42. Bryant MP \& Robinson IM (1961) An improved nonselective culture medium for ruminal bacteria and its use in determining diurnal variation in numbers of bacteria in the rumen. J Dairy Sci 44, 1446-1456.

43. Caldwell DR \& Bryant MP (1966) Medium without rumen fluid for nonselective enumeration and isolation of rumen bacteria. Appl Microbiol 14, 794-801.

44. Miller LT (1982) Single derivatization method for routine analysis of bacterial whole-cell fatty acid methyl esters, including hydroxyl acids. J Clin Microbiol 16, 584-586.

45. Abnous K, Brooks SPJ, Kwan J, et al. (2009) Diets enriched in oat bran or wheat bran temporally and differentially alter the composition of the fecal community of rats. J Nutr $\mathbf{1 3 9}$, 2024-2031.

46. Lane DJ (1991) 16S/23S rRNA sequencing. In Nucleic Acid Techniques in Bacterial Systematics, pp. 115-175 [E Stackebrandt and M Goodfellow, editors]. New York: John Wiley \& Sons.

47. Costa E, Puhl NJ, Selinger LB, et al. (2009) Characterization of mucosa-associated bacterial communities of the mouse intestine by terminal restriction fragment length polymorphism: utility of sampling strategies and methods to reduce single-stranded DNA artifacts. J Microbiol Meth 78, 175-180

48. Culman SW, Bukowski R, Gauch HG, et al. (2009) T-REX: software for the processing and analysis of T-RFLP data. BMC Bioinformatics 10, 171

49. Anderson M.J (2001) A new method for non-parametric multivariate analysis of variance. Austral Ecol 26, 32-46.

50. Kalmokoff M, Zwicker B, O'Hara M, et al. (2013) Temporal change in the gut community of rats fed high amylose cornstarch is driven by endogenous urea rather than strictly on carbohydrate availability. J Appl Microbiol 114, 1516-1528.

51. Schloss PD, Westcott SL, Ryabin T, et al. (2009) Introducing mothur: open-source, platform-independent, communitysupported software for describing and comparing microbial communities. Appl Environ Microbiol 75, 7537-7541.

52. Sun Y, Cai Y, Liu L, et al. (2009) Esprit: estimating species richness using large collections of 16s rRNA pyrosequences. Nucleic Acids Res 37, e76-e76.

53. Quast C, Pruesse E, Yilmaz P, et al. (2013) The SILVA ribosomal RNA gene database project: improved data processing and web-based tools. Nucleic Acids Res 41, D590-D596.

54. Gouy M, Guindon S \& Gascuel O (2010) SeaView version 4: a multiplatform graphical user interface for sequence alignment and phylogenetic tree building. Mol Biol Evol 27, 221-224.

55. Cole JR, Chai B, Marsh TL, et al. (2003) The ribosomal database project (rdp-II): Previewing a new autoaligner that allows regular updates and the new prokaryotic taxonomy. Nucleic Acids Res 31, 442-443.

56. Huson DH, Auch AF, Qi J, et al. (2007) MEGAN analysis of metagenomic data. Genome Res 17, 377-386.

57. Cantarel BL, Coutinho PM, Rancurel C, et al. (2009) The Carbohydrate-Active EnZymes database (CAZy): an expert resource for Glycogenomics. Nucleic Acids Res 37, D233-D238.
58. McCune B \& Grace JB (2002) Analysis of Ecological Communities. Gleneden Beach, OR: MjM Software Design.

59. Buttigieg PL \& Ramette A (2014) A guide to statistical analysis in microbial ecology: a community-focused, living review of multivariate data analyses. FEMS Microbiol Ecol $\mathbf{9 0}$, 543-550.

60. Mielke PW Jr, Berry KJ \& Johnson ES (1976) Multi-response permutation procedures for a priori classifications. Commun Stat Theory Method 5, 1409-1424.

61. Chao A \& Lee SM (1992) Estimating the number of classes via sample coverage. J Am Stat Assoc 87, 210-217.

62. Kurokawa K, Itoh T, Kuwahara T, et al. (2007) Comparative metagenomics revealed commonly enriched gene sets in human gut microbiomes. DNA Res 14, 169-181.

63. Thomas MC, Thomas DK, Kalmokoff ML, et al. (2012) Molecular methods to measure intestinal bacteria: a review. J AOAC Int 95, 5-23.

64. Stoddard SF, Smith BJ, Hein R, et al. (2015) rrnDB: improved tools for interpreting rRNA gene abundance in bacteria and archaea and a new foundation for future development. Nucleic Acids Res 43, D593-D598.

65. Kleessen B, Schwarz S, Boehm A, et al. (2007) Jerusalem artichoke and chicory inulin in bakery products affect faecal microbiota of healthy volunteers. Br J Nutr 98, 540-549.

66. Shastri P, McCarville J, Kalmokoff M, et al. (2015) Sex differences in gut fermentation and immune parameters in rats fed an oligofructose-supplemented diet. Biol Sex Differ 6, 13.

67. Faith JJ, McNulty NP, Rey FE, et al. (2011) Predicting a human gut microbiota's response to diet in gnotobiotic mice. Science 333, 101-104.

68. Kalmokoff M, Franklin J, Pertronella N, et al. (2015) Phylum level change in the cecal and fecal gut communities of rats fed diets containing different fermentable substrates supports a role for nitrogen as a factor contributing to community structure. Nutrients 7, 3279-3299.

69. Schneider ARJ, Jepp K, Murczynski L, et al. (2007) The inulin hydrogen breath test accurately reflects orocaecal transit time. Eur J Clin Invest 37, 802-807.

70. Macfarlane GT, Gibson GR \& Cummings JH (1992) Comparison of fermentation reactions in different regions of the human colon. J Appl Bacteriol 72, 57-64.

71. Kleessen B, Hartmann L \& Blaut M (2003) Fructans in the diet cause alterations of intestinal mucosal architecture, released mucins and mucosa-associated bifidobacteria in gnotobiotic rats. Br J Nutr 89, 597-606.

72. Ten Bruggencate SJM, Bovee-Oudenhoven IMJ, LettinkWissink MLG, et al. (2003) Dietary fructo-oligosaccharides dose-dependently increase translocation of salmonella in rats. J Nutr 133, 2313-2318.

73. Ten Bruggencate SJM, Bovee-Oudenhoven IMJ, LettinkWissink MLG, et al. (2004) Dietary fructo-oligosaccharides and inulin decrease resistance of rats to salmonella: protective role of calcium. Gut 53, 530-535.

74. Ten Bruggencate SJM, Bovee-Oudenhoven IMJ, LettinkWissink MLG, et al. (2005) Dietary fructooligosaccharides increase intestinal permeability in rats. $J$ Nutr 135, 837-842.

75. Tailford LE, Crost EH, Kavanaugh D, et al. (2015) Mucin glycan foraging in the human gut microbiome. Front Genet $\mathbf{6}, 81$.

76. Milani C, Turroni F, Duranti S, et al. (2016) Genomics of the genus Bifidobacterium reveals species-specific adaptation to the glycan-rich gut environment. Appl Environ Microbiol 82, 980-991.

77. Ruas-Madiedo P, Gueimonde M, Fernández-García M, et al. (2008) Mucin degradation by Bifidobacterium strains isolated from the human intestinal microbiota. Appl Environ Microbiol 74, 1936-1940. 
78. Salyers AA, Vercellotti JR, West SE, et al. (1977) Fermentation of mucin and plant polysaccharides by strains of Bacteroides from the human colon. Appl Environ Microbiol 33, 319-322.

79. Lopez-Siles M, Khan TM, Duncan SH, et al. (2012) Cultured representatives of two major phylogroups of human colonic Faecalibacterium prausnitzii can utilize pectin, uronic acids, and host-derived substrates for growth. Appl Environ Microbiol 78, 420-428.

80. Salonen A, Lahti L, Salojärvi J, et al. (2014) Impact of diet and individual variation on intestinal microbiota composition and fermentation products in obese men. ISME $J \mathbf{8}$, 2218-2230.
81. Zweigner J, Schumann RR \& Weber JR (2006) The role of lipopolysaccharide-binding protein in modulating the innate immune response. Microb Infect 8, 946-952.

82. Pineiro M, Asp NG, Reid G, et al. (2008) FAO Technical meeting on prebiotics. J Clin Gastroenterol 42, S156-S159.

83. Brooks SPJ \& Kalmokoff ML (2012) Prebiotics and probiotics: some thoughts on demonstration of efficacy within the regulatory sphere. $J A O A C$ Int $\mathbf{9 5}, 2-4$.

84. Van den Abbeele P, Gérard P, Rabot S, et al. (2011) Arabinoxylans and inulin differentially modulate the mucosal and luminal gut microbiota and mucin-degradation in humanized rats. Environ Microbiol 13, 2667-2680. 